\title{
O papel do exercício físico na prevenção das quedas nos idosos: uma revisão baseada na evidência
}

Patrícia Cunha,* Luísa Costa Pinheiro*

\section{RESUMO}

Objetivos: As quedas, definidas pela Organização Mundial da Saúde como uma passagem não intencional para o chão ou outro nível inferior àquele em que a pessoa se encontra, não são acontecimentos ao acaso, podendo ser previstas através da avaliação de fatores de risco. A população idosa encontra-se mais vulnerável, estimando-se que $30 \%$ das pessoas com 65 ou mais anos caiam, pelo menos, uma vez por ano, representando uma importante causa de incapacidade e elevados custos para a saúde, potencialmente evitáveis. Pretende-se, com este trabalho, determinar o efeito do exercício físico (EF) na prevenção das quedas nos idosos. Fontes de dados: Bases de dados MEDLINE e sítios de medicina baseada na evidência.

Métodos de revisão: Pesquisa de normas de orientação clínica, metanálises (MA), revisões sistemáticas (RS) e ensaios clínicos aleatorizados e controlados, publicados entre janeiro de 2010 e setembro de 2014, utilizando os termos MeSH: ACCIDENTAL FALLS, ACCIDENT PREVENTION, AGED e EXERCISE. Foi utilizada a Strength of Recommendation Taxonomy (SORT) para a atribuição dos níveis de evidência e forças de recomendação.

Resultados: Foram encontrados 203 artigos. Apenas cinco preencheram os critérios de inclusão: três MA e duas RS. Há evidência de que os programas de exercícios para prevenção de quedas em pessoas mais velhas não só reduzem a taxa de quedas, mas também evitam lesões decorrentes das mesmas. Muitos dos fatores de risco para quedas são corrigíveis por programas de exercícios bem desenhados e parecem igualmente eficazes se praticados no domicílio. O tipo de EF mais eficaz na prevenção das quedas nos idosos é o treino de equilíbrio e os programas de EF são mais eficazes se prolongados no tempo e de maior intensidade (maior número de horas e pelo menos duas vezes por semana).

Conclusões: De acordo com a evidência disponível, os programas de EF devem ser recomendados porque são eficazes na diminuição do número e risco de quedas nos idosos (Força de recomendação A).

Palavras-chave: Acidentes por Quedas; Prevenção de Acidentes; Idoso; Exercício.

\section{INTRODUÇÃO}

A s quedas, definidas como escorregamento ou tropeçamento que resulta em ferimentos, não são acontecimentos ao acaso..$^{1-2}$ A população idosa portuguesa, seguindo a tendência mundial, tem vindo a aumentar. ${ }^{3}$ Com o envelhecimento surgem inúmeras mudanças físicas, sensoriais e cognitivas que diminuem a eficiência, a aptidão para se adaptar e a funcionalidade, algo inevitável, manifes-

* Médicas Internas de Medicina Geral e Familiar

Unidade de Saúde Familiar de Fiães, ACES Entre Douro e Vouga I - Feira/Arouca. tando-se ao longo do processo natural e complexo que é o envelhecimento e levando a um aumento do risco de queda e lesões decorrentes de uma queda.

Estima-se que $30 \%$ das pessoas com 65 ou mais anos caiam, pelo menos, uma vez por ano. ${ }^{4}$ As quedas são a maior causa de acidentes e de compromisso da condição de saúde das pessoas idosas, representando um sério problema de saúde pública. ${ }^{5}$ Constituem a principal razão de admissão aos serviços de urgência neste grupo etário, assim como um dos principais fatores precipitantes da institucionalização desta população. ${ }^{6}$

Entre as repercussões da idade sobre a função, que 
podem levar à queda da pessoa idosa, destacam-se: diminuição da força muscular, dos reflexos, da flexibilidade, da velocidade espontânea da marcha, acuidade visual e função vestibular. Nesse sentido, observa-se que as quedas são causas importantes de morbilidade entre os idosos e podem ter consequências desastrosas. Além do risco de fraturas, há perda de confiança para caminhar, devido ao temor de novas quedas, fazendo o idoso diminuir a sua mobilidade, formando-se um círculo vicioso, pois com a restrição de atividades há diminuição da força muscular, enfraquecimento dos membros inferiores, levando à condição de dependência e isolamento social. ${ }^{7}$ As quedas representam, desta forma, elevados custos para a saúde, que são potencialmente evitáveis, uma vez que o risco de quedas é multifatorial. ${ }^{8}$

A prevenção das quedas é eficaz, podendo ser elaboradas estratégias para modificar ou eliminar aqueles fatores passíveis de atuação, pelo que cabe ao médico de família (MF) identificar a população-alvo e promover a implementação de medidas preventivas. O exercício físico (EF) pode ser uma dessas medidas? Para responder a esta questão, foi feita uma revisão baseada na evidência sobre o benefício do EF na prevenção das quedas nos idosos.

\section{METODOLOGIA}

Foi realizada uma pesquisa bibliográfica entre $15 \mathrm{e}$ 20 de setembro de 2014, nas seguintes bases de dados: National Guideline Clearinghouse, Canadian Medical Association Practice Guidelines Infobase, Cochrane Library, DARE, Bandolier, Índex de revistas médicas portuguesas e PubMed. Foram pesquisadas normas de orientação clínica, metanálises (MA), revisões sistemáticas (RS) e ensaios clínicos aleatorizados e controlados (ECAC) em português, espanhol e inglês, publicados entre janeiro de 2010 e setembro de 2014, utilizando os termos MeSH ACCIDENTAL FALLS, ACCIDENT PREVENTION, AGED e EXERCISE.

Foi definido que os estudos deveriam cumprir critérios de inclusão que respondessem às quatro questões PICO:

- População: Idosos (indivíduos com 65 ou mais anos de idade).

- Intervenção: Prática de exercício físico (qualquer tipo).

- Comparação: Placebo ou ausência de atividade física.
- Resultados: número e/ou taxa de quedas.

Na avaliação dos estudos e atribuição dos níveis de evidência (NE) e forças de recomendação foi utilizada a escala Strength of Recommendation Taxonomy (SORT), da American Family Physician.

\section{RESULTADOS}

Da pesquisa obtiveram-se 203 artigos. Destes, cinco cumpriram os critérios de inclusão: três MA e duas RS. Foram excluídos os artigos repetidos, os não concordantes com os objetivos ou os que não cumpriam os critérios de inclusão. Também foram excluídos os ensaios clínicos controlados e aleatorizados incluídos nas MA e RS.

A descrição dos artigos incluídos encontra-se resumida nos Quadros I e II.

A MA de Thomas et al, ${ }^{10}$ publicada em 2011, incluiu sete ensaios clínicos num total de 1.503 idosos. A intervenção proposta constava no Programa de Exercícios de Otago (PEO), constituído por um conjunto de exercícios de fortalecimento muscular da perna, exercícios de equilíbrio e um plano de caminhada, comparado com um grupo controlo, com ausência de EF. No PEO, os exercícios são prescritos individualmente e aumentam em dificuldade durante uma série de cinco visitas domiciliares por um instrutor treinado. Os exercícios demoram cerca de 30 minutos e são realizados três vezes por semana e a caminhada é realizada, pelo menos, duas vezes por semana. Concluiu-se que o grupo de idosos que praticava o $\mathrm{PEO}$ reduziu significativamente a taxa de quedas (odds ratio $(\mathrm{OR})=0,45$; intervalo de confiança (IC) $95 \%=0,25-0,80$ ). A este estudo, os autores da presente revisão atribuíram um NE 1.

A MA de El-Khoury et al, ${ }^{11}$ data de 2013 e incluiu 17 ensaios clínicos, num total de 4.305 idosos. Compara a prática de EF ( $n=2.195)$ versus a ausência do mesmo $(n=2.110)$. Em 14 dos ECAC, a prática de EF é realizada em grupo, sendo que em seis destes 14 há também sessões de EF em casa. Nos restantes três ECAC, as sessões de EF são individualizadas e apenas realizadas no domicílio. Concluem haver evidência de que os programas de exercícios para prevenção de quedas em pessoas mais velhas não só reduzem as taxas de quedas (OR=0,63; IC 95\%=0,51-0,77), mas também evitam lesões decorrentes das mesmas (OR=0,57; IC 95\%=0,360,90 ), pelo que muitos dos fatores de risco para quedas são corrigíveis por programas de exercícios bem dese- 


\begin{tabular}{|c|c|c|c|c|}
\hline Referência & População & Intervenção & Resultados & NE \\
\hline $\begin{array}{l}\text { Thomas et al }{ }^{10} \\
\text { (2011) }\end{array}$ & $\begin{array}{l}7 \text { ECAC } \\
\mathrm{n}=1.503 \\
\text { idosos }\end{array}$ & $\begin{array}{l}\text { Programa de exercícios de Otago } \\
\text { vs. } \\
\text { Ausência de EF }\end{array}$ & $\begin{array}{l}\text { O Programa de exercícios de Otago reduz } \\
\text { significativamente a taxa de quedas nos idosos } \\
(\mathrm{OR}=0,45 ; \text { IC } 95 \%=0,25-0,80)\end{array}$ & 1 \\
\hline $\begin{array}{l}\text { El-Khoury } \\
\text { et } a l^{11} \\
(2013)\end{array}$ & $\begin{array}{l}17 \text { ECAC } \\
n=4.305 \\
\text { idosos }\end{array}$ & $\begin{array}{l}\mathrm{EF}(\mathrm{n}=2.195) \\
\text { vs. } \\
\text { Ausência de EF }(n=2.110)\end{array}$ & $\begin{array}{l}\text { - Os programas de EF para prevenção de quedas em } \\
\text { idosos não só reduzem as taxas de quedas (OR=0,63; } \\
\text { IC } 95 \%=0,51-0,77) \text {, mas também evitam lesões } \\
\text { decorrentes de quedas (OR=0,57; IC } 95 \%=0,36-0,90) \\
\text { - Muitos dos fatores de risco para quedas são corrigíveis } \\
\text { por programas de exercícios bem desenhados }\end{array}$ & 1 \\
\hline $\begin{array}{l}\text { Sherrington } \\
\text { et } a l^{12} \\
(2011)\end{array}$ & $\begin{array}{l}54 \text { ECAC } \\
n=26.102 \\
\text { idosos }\end{array}$ & $\begin{array}{l}\text { EF } \\
\text { vs. } \\
\text { Ausência de EF }\end{array}$ & $\begin{array}{l}\text { - O EF reduz em } 16 \% \text { a taxa de quedas nos idosos } \\
\text { (OR=0,84; IC } 95 \%=0,77-0,91) \\
\text { - O treino de equilíbrio em associação com treino em } \\
\text { doses elevadas de exercício ( }>2 \mathrm{~h} / \text { semana; }>6 \text { meses) } \\
\text { reduziram em } 38 \% \text { a taxa de quedas (OR=0,64; } \\
\text { IC } 95 \%=0,40-1,02 \text { ) }\end{array}$ & 1 \\
\hline
\end{tabular}

Legenda: ECAC - Ensaios clínicos aleatorizados e controlados; EF - Exercício físico; OR - Odds ratio; IC - Intervalo de confiança

\section{QUADRO II. Revisões sistemáticas}

\begin{tabular}{|c|c|c|c|c|}
\hline Referência & População & Intervenção & Resultados & NE \\
\hline $\begin{array}{l}\text { Martin et al }{ }^{13} \\
\text { (2013) }\end{array}$ & $\begin{array}{l}10 \text { ECAC } \\
n=2.293 \\
\text { idosos }\end{array}$ & $\begin{array}{l}2 \text { grupos: } \\
\text { 1) EF/Fisioterapia } \\
\text { vs. } \\
\text { Ausência de EF (7 ECAC) } \\
\text { 2) EF/Fisioterapia } \\
\text { vs. } \\
\text { Programas de exercícios no } \\
\text { domicílio (3 ECAC) }\end{array}$ & $\begin{array}{l}\text { 1) Comparado com os idosos sem EF: diminuição } \\
\text { significativa no número de quedas }(p=0,02) \\
\text { 2) Comparado com o grupo com programa de exercícios } \\
\text { no domicílio: } 2 \text { ECAC não mostraram diferenças } \\
\text { significativas entre ambas intervenções }(p=0,87)\end{array}$ & 2 \\
\hline $\begin{array}{l}\text { Michael et al }{ }^{14} \\
(2010)\end{array}$ & $\begin{array}{l}18 \text { ECAC } \\
n=3.986 \\
\text { idosos }\end{array}$ & $\begin{array}{l}\text { EF/Fisioterapia } \\
\text { vs. } \\
\text { Ausência de EF }\end{array}$ & $\begin{array}{l}\text { O EF/Fisioterapia reduz o risco de cair em } 13 \% \\
(\mathrm{OR}=0,85 ; \mathrm{IC} 95 \%=0,78-0,92) \text { e treinos mais intensos } \\
\text { (>n. de horas) estão associados a maiores reduções do } \\
\text { risco de queda }(\mathrm{OR}=0,87 ; \mathrm{IC} 95 \%=0,81-0,94)\end{array}$ & 2 \\
\hline
\end{tabular}

Legenda: ECAC - Ensaios clínicos aleatorizados e controlados; EF - Exercício físico; OR - Odds ratio; IC - Intervalo de confiança

nhados. A esta MA as autoras atribuíram um NE 1.

A MA de Sherrington et al, ${ }^{12}$ publicada em 2011, engloba 54 ECAC ( $n=26.102$ idosos) que comparam a prática de EF com um grupo controlo (ausência de EF). No grupo que realiza EF comprovou-se uma redução significativa ( $16 \%$ ) na taxa de quedas nos idosos (OR=0,84; IC $95 \%=0,77-0,91)$. O treino de equilíbrio em associação com treino de exercício, durante mais de $2 \mathrm{~h} /$ semana e mais de seis meses, promoveu uma redução não significativa de $38 \%$ na taxa de quedas $(\mathrm{OR}=0,64$; IC 95\% $=0,40$ 1,02). A esta MA as autoras atribuíram um NE 1.

A RS de Martin et al, ${ }^{13}$ de 2013, incluiu 10 ensaios clínicos, num total de 2.293 idosos. Existiam dois grupos: 1) EF/Fisioterapia versusgrupo controlo (ausência de EF 
e de fisioterapia) e 2) EF/Fisioterapia versus programas de EF no domicílio, com sete e três ensaios, respetivamente. No primeiro grupo, todos os estudos relataram uma diminuição significativa no número de quedas no grupo de intervenção em comparação com o grupo controlo ( $p=0,02 ; \mathrm{RR}=0,71$; IC $95 \%=0,60-0,82$ ). No segundo grupo, dois dos três ensaios não mostraram diferenças significativas entre as intervenções $(p=0,87)$, pelo que se concluiu que a prática de EF no domicílio seria tão benéfica quanto o exercício praticado em grupo ou por um terapeuta. Os estudos incluídos nesta RS apresentam heterogeneidade clínica e estatística $\left(\mathrm{I}^{2}=61,5 \%\right)$, pelo que lhe foi atribuído um NE 2.

Por último, a RS de Michael YL et al, ${ }^{14}$ publicada em 2010, inclui 18 ensaios clínicos, num total de 3.986 idosos. Na comparação da realização de EF/Fisioterapia versus controlo verificou-se diminuição de $13 \%$ do risco de quedas no primeiro grupo $(\mathrm{OR}=0,85$; IC $95 \%=0,78-0,92)$. Sugere-se ainda que treinos mais intensos (maior número de horas) estão associados a reduções pequenas, mas estatisticamente significativas (OR=0,87; IC $95 \%=0,81$ $0,94)$, do risco de quedas. Pela qualidade limitada dos estudos, sem referência a dupla ocultação/controlo dos ensaios, as autoras atribuíram um NE 2.

\section{CONCLUSÃO}

Os programas de EF, isoladamente ou associados a outras intervenções multifatoriais, são eficazes na diminuição do número e risco de quedas nos idosos (NE 1).

De acordo com a evidência disponível, o EF deve ser recomendado para a população de idosos em geral (SORT A). Nos idosos com maior risco de quedas verificou-se uma maior redução deste risco, pelo que se conclui que estes serão os que mais beneficiam da prática de EF para redução da taxa de quedas (NE 1). ${ }^{12}$

Os programas de EF demonstraram ser igualmente eficazes se praticados no domicílio (NE 2). ${ }^{13}$ Os programas com intervenções em grupo e realizadas em ambientes alheios ao quotidiano, podem ter a dificuldade em realizar um programa, simultaneamente motivador e seguro para todos os participantes. É fundamental que os exercícios possam ser individualizados, orientados $\mathrm{e}$ modificados de forma a minimizar as dificuldades e maximizar as capacidades dos intervenientes. ${ }^{11-12}$

No âmbito da prescrição de exercício, aconselhamento e supervisão de programas individuais ou em grupo é fundamental considerar os aspetos motivacionais para a mudança de comportamento que possam contribuir para a adesão dos participantes, assim como para a efetividade das abordagens. ${ }^{11}$

O tipo de EF mais eficaz na prevenção das quedas nos idosos é o treino de equilíbrio. Mudanças moderadas a elevadas do equilíbrio, após um programa centrado no treino de equilíbrio, conduzem a um significativo efeito protetor na redução de quedas (NE 1). ${ }^{12}$

O EF é mais eficaz se prolongado no tempo e de maior intensidade - maior número de horas e pelo menos duas vezes por semana (NE 1). ${ }^{12,14}$

Para além do assinalado, a prática de EF tem outras vantagens, como favorecer o convívio e o desenvolvimento psicossocial dos seus participantes, não ter custos elevados para o SNS e ser possível de aplicar na comunidade, onde o MF tem um papel preponderante no seu estímulo e proposta.

Como limitações às conclusões desta revisão, é importante referir a grande heterogeneidade dos programas de EF quanto à descrição específica da frequência, da intensidade, duração e progressão do EF. Importa também salientar que as conclusões refletem efeitos nas populações a que se destinam, pelo que os programas de EF devem estar sempre adaptados ao indivíduo a quem vão ser aplicados, tendo em conta as suas características particulares.

É importante incentivar a implementação desta medida preventiva por equipas multidisciplinares na comunidade, não esquecendo que a abordagem holística do MF pode incidir complementarmente na prevenção de outros fatores de risco para quedas, como as alterações da visão, da audição, entre outras.

\section{REFERÊNCIAS BIBLIOGRÁFICAS}

1. World Health Organization. WHO global report on falls revention in older age [Internet]. Geneva:WHO; 2007. ISBN 9789241563536. Available from: http://www.who.int/ageing/publications/Falls_prevention7March.pdf

2. Ganz DA, BaoY, Shekelle PG, Rubenstein LZ.Will my patient fall? JAMA. 2007;297(1):77-86.

3. Instituto Nacional de Estatística. Anuário estatístico de Portugal, 2013. Lisboa:INE; 2014.Available from: http://www.ine.pt/xportal/xmain?xpid $=$ INE\&xpgid=ine_destaques\&DESTAQUESdest_boui=223549784\&DE STAQUESmodo $=2$

4. Gillespie LD, Robertson MC, Gillespie WJ, Sherrington C, Gates S, Clemson LM, et al. Interventions for preventing falls in older people living in the community. Cochrane Database Syst Rev. 2012 Sep 12;9: 
CD007146.

5. Sjösten NM, Salonoja M, Piirtola M, Vahlberg T, Isoaho R, Hyttinen $H$, et al. A multifactorial fall preventin programme in home-dwelling elderly people: a randomized-controlled trial. Public Health. 2007;121(4):308-18.

6. National Institute for Clinical Excellence. Falls: the assessment and prevention of falls in older people: NICE clinical guideline 21 [This guideline has been updated and replaced by NICE guideline CG161]. London: NICE; 2013 [updated 2016 Jan]. Available from: https://www.nice. org.uk/guidance/cg161

7. Lundin-Olsson L. Community-dwelling older adults with balance impairment show a moderate increase in fall risk, although further research is required to refine how balance measurement can be used in clinical practice. Evid Based Nurs. 2010;13(3):96-7.

8. Davis JC, Robertson MC, Ashe MC, Liu-Ambrose T, Khan KM, Marra CA. International comparison of cost of falls in older adults living in the community: a systematic review. Osteoporos Int. 2010;21(8):1295-306.

9. Rubenstein LZ. Falls in older people: epidemiology, risk factors and strategies for prevention. Age Ageing. 2006;35 Suppl 2:ii37-ii41.

10. Thomas S, Mackintosh S, Halbert J. Does the 'Otago exercise programme' reduce mortality and falls in older adults? A systematic review and meta-analysis. Age Ageing. 2010;39(6):681-7.

11. El-Khoury F, Cassou B, Charles MA, Dargent-Molina P. The effect of fall prevention exercise programmes on fall induced injuries in communi- ty dwelling older adults: systematic review and meta-analysis of randomised controlled trials. BMJ. 2013;347:f6234.

12. Sherrington C, Tiedemann A, Fairhall N, Close JC, Lord SR. Exercise to prevent falls in older adults: an update meta-analysis and best practice recommendation. N SW Public Health Bull. 2011;22(3-4):78-83.

13. Martin JT, Wolf A, Moore JL, Rolenz E, DiNinno A, Reneker JC. The effectiveness of physical therapist-administered group-based exercise on fall prevention: a systematic review of randomized controlled trials. J Geriatr Phys Ther. 2013;36(4):182-93.

14. Michael YL, Whitlock EP, Lin JS, Fu R, O'Connor EA, Gold R, et al. Primary care - relevant interventions to prevent falling in older adults: $a$ systematic evidence review for the U.S. preventive services task force. Ann Intern Med. 2010;153(12):815-25.

\section{CONFLITO DE INTERESSES}

As autoras declaram não ter conflitos de interesses.

\author{
ENDEREÇO PARA CORRESPONDÊNCIA \\ Patrícia Cunha \\ R. Amândio Galhano, n. ${ }^{\circ} 33$, Apartamento 1.2, 4200-005 Porto \\ E-mail: patriciarodriguescunha@gmail.com
}

Recebido em 08-03-2015

Aceite para publicação em 01-02-2016

\section{ABSTRACT}

\section{THE ROLE OF PHYSICAL EXERCISE IN THE PREVENTION OF FALLS IN THE ELDERLY: AN EVIDENCE BASED REVIEW}

Objectives: Falls are defined as events in which a person comes to rest inadvertently on the ground or floor or other lower level. They do not happen by chance, but can be predicted through the assessment of risk factors. The elderly population is more vulnerable. It is estimated that $30 \%$ of people aged 65 or more fall at least once a year. This represents a potentially preventable cause of disability and high health care costs. The aim of this study is to determine the benefits of physical exercise (PE) in the prevention of falls in the elderly.

Data sources: MEDLINE and evidence-based medicine databases.

Review methods: Research of clinical guidelines, meta-analyses (MA), systematic reviews (SR) and randomized controlled clinical trials, published between January 2010 and September 2014, using the MeSH terms: ACCIDENTAL FALLS, ACCIDENT PREVENTION, AGED and EXERCISE. Strength of Recommendation Taxonomy (SORT) was utilized for the assignment of levels of evidence and strength of recommendations.

Results: We found 203 articles. Five met the inclusion criteria, including three MA and two SR. There is evidence that exercise programs to prevent falls in older people not only reduce the incidence of falls but also prevent injuries arising from falls. Many of the risk factors for falls are correctable by well-designed exercise programs. These are equally effective if practiced at home. The most effective type of PE in the prevention of falls in the elderly is balance training. PE programs are more effective if prolonged in time and of greater intensity (a greater number of hours and at least twice a week).

Conclusions: PE programs should be recommended because they are effective in decreasing the number and risk of falls in the elderly (Strength of Recommendation A).

Keywords: Accidental Falls; Accident Prevention; Aged; Exercise. 